\title{
From Bi-ideals to Periodicity
}

\author{
Jānis Buls, Aivars Lorencs \\ Department of Mathematics, University of Latvia, Raiņa bulvāris 19, \\ Rīga, LV-1586, Latvia \\ buls@fmf.lu.lv
}

28.07 .06

\begin{abstract}
The necessary and sufficient conditions are extracted for periodicity of bi-ideals. By the way two proper subclasses of uniformly recurrent words are introduced.
\end{abstract}

\section{Introduction}

The periodicities are fundamental objects, due to their primary importance in word combinatorics [8,9] as well as in various applications. The study of periodicities is motivated by the needs of molecular biology [6] and computer science. Particularly, we mention here such fields as string matching algorithms [4], text compression [14] and cryptography [12].

In different areas of mathematics, people consider a lot of hierarchies which are typically used to classify some objects according to their complexity. Here we deal with hierarchy

$$
\mathfrak{B} \supset \mathfrak{R}_{\mathfrak{u}} \supset \mathfrak{P}, \quad \text { where }
$$

$\mathfrak{B}$ - the class of bi-ideals,

$\mathfrak{R}_{\mathfrak{u}}$ - the class of uniformly recurrent words,

$\mathfrak{P}$ - the class of periodic words.

This hierarchy comes from combinatorics on words, where these classes are being investigated intensively (cf. $[2,8,9,10]$ ). Bi-ideal sequences have been considered, with different names, by several authors in algebra and combinatorics $[1,3,7,13,17]$.

We refine the hierarchy $\mathfrak{B} \supset \mathfrak{R}_{\mathfrak{u}} \supset \mathfrak{P}$ to the chain

$$
\mathfrak{B} \supset \mathfrak{R}_{\mathfrak{u}} \supset \mathfrak{B}_{\mathfrak{b}} \supset \mathfrak{B}_{\mathfrak{f}} \supset \mathfrak{P}, \quad \text { where }
$$

$\mathfrak{B}_{\mathfrak{b}}$ — the class of bounded bi-ideals,

$\mathfrak{B}_{\mathfrak{f}}$ — the class of finitely generated bi-ideals. So we localize the class of uniformly recurrent words by means of bi-ideals. Corollary 7 gives one method how the words of $\mathfrak{B}_{\mathfrak{f}}$ can be generated.

At first we characterize periodic finitely generated bi-ideals: we give one necessary condition [Corollary 8] in prefix-suffix terms and demonstrate this is not sufficient [Example 12]. Then we turn our attention to factors and prove sufficient and necessary condition [Theorem 21], and demonstrate this is not necessary for bounded bi-ideals [Example 34]. Lastly we extract exhaustive description [Theorem 37] of periodicity for all class of bi-ideals (more complicated of course). 


\section{Preliminaries}

In this section we present most of the notations and terminology used in this paper. Our terminology is more or less standard (cf. [10]) so that a specialist reader may wish to consult this section only if need arise.

Let $A$ be a finite non-empty set and $A^{*}$ the free monoid generated by $A$. The set $A$ is also called an alphabet, its elements letters and those of $A^{*}$ finite words. The role of identity element is performed by empty word and denoted by $\lambda$. We set $A^{+}=A^{*} \backslash\{\lambda\}$.

A word $w \in A^{+}$can be written uniquely as a sequence of letters as $w=w_{1} w_{2} \ldots w_{l}$, with $w_{i} \in A$, $1 \leq i \leq l, l>0$. The integer $l$ is called the length of $w$ and denoted $|w|$. The length of $\lambda$ is 0 . We set $w^{0}=\lambda \wedge \forall i w^{i+1}=w^{i} w$

$$
w^{+}=\bigcup_{i=1}^{\infty}\left\{w^{i}\right\}, \quad w^{*}=w^{+} \cup\{\lambda\} .
$$

A positive integer $p$ is called a period of $w=w_{1} w_{2} \ldots w_{l}$ if the following condition is satisfied:

$$
1 \leq i \leq l-p \Rightarrow w_{i}=w_{i+p} .
$$

We recall the important periodicity theorem due to Fine and Wilf [5]:

Theorem 1. Let $w$ be a word having periods $p$ and $q$ and denote by $\operatorname{gcd}(p, q)$ the greatest common divisor of $p$ and $q$. If $|w| \geq p+q-\operatorname{gcd}(p, q)$, then $w$ has also the $\operatorname{period} \operatorname{gcd}(p, q)$.

The word $w^{\prime} \in A^{*}$ is called a factor (or subword) of $w \in A^{*}$ if there exist $u, v \in A^{*}$ such that $w=u w^{\prime} v$. The word $u$ (respectively $v$ ) is called a prefix (respectively a suffix) of $w$. The ordered triple $\left(u, w^{\prime}, v\right)$ is called an occurrence of $w^{\prime}$ in $w$. The factor $w^{\prime}$ is called proper factor if $w \neq w^{\prime}$. We denote respectively by $\mathrm{F}(w), \operatorname{Pref}(w)$ and $\operatorname{Suff}(w)$ the sets of $w$ factors, prefixes and suffixes.

An (indexed) infinite word $x$ on the alphabet $A$ is any total map $x: \mathbb{N} \rightarrow A$. We set for any $i \geq 0$, $x_{i}=x(i)$ and write

$$
x=\left(x_{i}\right)=x_{0} x_{1} \ldots x_{n} \ldots
$$

The set of all the infinite words over $A$ is denoted by $A^{\omega}$.

The word $w^{\prime} \in A^{*}$ is a factor of $x \in A^{\omega}$ if there exist $u \in A^{*}, y \in A^{\omega}$ such that $x=u w^{\prime} y$. The word $u$ (respectively $y$ ) is called a prefix (respectively a suffix) of $x$. We denote respectively by $\mathrm{F}(x), \operatorname{Pref}(x)$ and $\operatorname{Suff}(x)$ the sets of $x$ factors, prefixes and suffixes. For any $0 \leq m \leq n$, both $x[m, n]$ and $x[m, n+1)$ denote a factor $x_{m} x_{m+1} \ldots x_{n}$. The indexed word $x[m, n]$ is called an occurrence of $w^{\prime}$ in $x$ if $w^{\prime}=x[m, n]$. The suffix $x_{n} x_{n+1} \ldots x_{n+i} \ldots$ is denoted by $x[n, \infty)$.

If $v \in A^{+}$we denote by $v^{\omega}$ the infinite word $v^{\omega}=v v \ldots v \ldots$ This word $v^{\omega}$ is called a periodic word. The concatenation of $u=u_{1} u_{2} \ldots u_{k} \in A^{*}$ and $x \in A^{\omega}$ is the infinite word

$$
u x=u_{1} u_{2} \ldots u_{k} x_{0} x_{1} \ldots x_{n} \ldots
$$

A word $x$ is called ultimately periodic if there exist words $u \in A^{*}, v \in A^{+}$such that $x=u v^{\omega}$. In this case, $|u|$ and $|v|$ are called, respectively, an anti-period and a period.

A sequence of words of $A^{*}$

$$
v_{0}, v_{1}, \ldots, v_{n}, \ldots
$$

is called a bi-ideal sequence if $\forall i \geq 0\left(v_{i+1} \in v_{i} A^{*} v_{i}\right)$. The term "bi-ideal sequence" is due to the fact that $\forall i \geq 0\left(v_{i} A^{*} v_{i}\right)$ is a bi-ideal of $A^{*}$.

Corollary 2. Let $\left(v_{n}\right)$ be a bi-ideal sequence. Then

$$
v_{m} \in \operatorname{Pref}\left(v_{n}\right) \cap \operatorname{Suff}\left(v_{n}\right)
$$

for all $m \leq n$ 
A bi-ideal sequence $v_{0}, v_{1}, \ldots, v_{n}, \ldots$ is called proper if $v_{0} \neq \lambda$. In the following the term bi-ideal sequence will be referred only to proper bi-ideal sequences.

If $v_{0}, v_{1}, \ldots, v_{n}, \ldots$ is a bi-ideal sequence, then there exists a unique sequence of words

$$
u_{0}, u_{1}, \ldots, u_{n}, \ldots
$$

such that

$$
v_{0}=u_{0}, \quad \forall i \geq 0\left(v_{i+1}=v_{i} u_{i+1} v_{i}\right)
$$

\section{The Class of Finitely Generated Bi-ideals}

Let us consider the set $A^{\infty}=A^{*} \cup A^{\omega}$ and $u, v \in A^{\infty}$. Then $d(u, v)=0$ if $u=v$, otherwise

$$
d(u, v)=2^{-n},
$$

where $n$ is the length of the maximal common prefix of $u$ and $v$. It is called a prefix metric.

Let $v_{0}, v_{1}, \ldots, v_{n} \ldots$ be an infinite bi-ideal sequence, where $v_{0}=u_{0}$ and $\forall i \geq 0\left(v_{i+1}=v_{i} u_{i+1} v_{i}\right)$. Since for all $i \geq 0$ the word $v_{i}$ is a prefix of the next word $v_{i+1}$ the sequence $\left(v_{i}\right)$ converges, with respect to the prefix metric, to the infinite word $x \in A^{\omega}$

$$
x=v_{0}\left(u_{1} v_{0}\right)\left(u_{2} v_{1}\right) \ldots\left(u_{n} v_{n-1}\right) \ldots
$$

This word $x$ is called a bi-ideal. We say the sequence $\left(u_{i}\right)$ generates the bi-ideal $x$.

Let $x$ be an infinite word. A factor $u$ of $x$ is called recurrent if it occurs infinitely often in $x$. The word $x$ is called recurrent when any of its factors is recurrent.

Proposition 3. (see, e.g., [10]) A word $x$ is recurrent if and only if it is a bi-ideal.

Lemma 4. (see, e.g., [10]) Let $x \in A^{\omega}$ be an ultimately periodic word. If $x$ is recurrent, then $x$ is periodic.

Due to this lemma we can restrict ourselves. Therefore we investigate only the periodicity of bi-ideals and say nothing about ultimately periodicity.

Definition 5. Let $\left(u_{i}\right)$ generates a bi-ideal $x$. The bi-ideal $x$ is called finitely generated if $\exists m \forall i \forall j(i \equiv$ $\left.j(\bmod m) \Rightarrow u_{i}=u_{j}\right)$. We say in this situation $m$-tuple $\left(u_{0}, u_{1}, \ldots, u_{m-1}\right)$ generates the bi-ideal $x$.

Theorem 6. If $\bigcup_{i=0}^{m-1} \operatorname{Pref}\left(u_{i}\right)$ or $\bigcup_{i=0}^{m-1} \operatorname{Suff}\left(u_{i}\right)$ has at least two words with the same length then a bi-ideal generated by $\left(u_{0}, u_{1}, \ldots, u_{m-1}\right)$ is not periodic.

Proof. Let $x \in A^{\omega}$ be a bi-ideal generated by $\left(u_{0}, u_{1}, \ldots, u_{m-1}\right)$.

(i) Let $\bigcup_{i=0}^{m-1} \operatorname{Pref}\left(u_{i}\right)$ has at least two words with one and the same length. Then there exist $u_{i}, u_{j}$ such that $u a \in \operatorname{Pref}\left(u_{i}\right), u b \in \operatorname{Pref}\left(u_{j}\right)$, where $u \in A^{*}, a, b \in A$ and $a \neq b$.

Let $T_{0}=|u a|$ and $t>T_{0}$. Then we can choose $n$ so great that $\left|v_{n}\right| \geq t$, where $v_{n+1}=v_{n} u_{i} v_{n}$. Hence $v_{n} u a \in \operatorname{Pref}\left(v_{n+1}\right)$. Therefore $a=x_{s}$, where $s=\left|v_{n} u\right|$.

Since the tuple $\left(u_{0}, u_{1}, \ldots, u_{m-1}\right)$ generates the bi-ideal $x$ then $\exists k>n v_{k+1}=v_{k} u_{j} v_{k}$. Hence $v_{k} u b \in$ $\operatorname{Pref}\left(v_{k+1}\right)$. Therefore $b=x_{\sigma}$, where $\sigma=\left|v_{k} u\right|$. Since $k>n$ then $v_{n} \in \operatorname{Suff}\left(v_{k}\right)$. Thus $x_{s-t}=x_{\sigma-t}$ but $x_{s}=a \neq b=x_{\sigma}$. That means that $t$ is not a period of $x$.

(ii) Let $\bigcup_{i=0}^{m-1} \operatorname{Suff}\left(u_{i}\right)$ has at least two words with one and the same length. Then there exist $u_{i}, u_{j}$ such that $a u \in \operatorname{Suff}\left(u_{i}\right), b u \in \operatorname{Suff}\left(u_{i}\right)$, where $u \in A^{*}, a, b \in A$ and $a \neq b$.

Let $T_{0}=|a u|$ and $t>T_{0}$. Then we can choose $n$ so great that $\left|v_{n}\right| \geq t$, where $v_{n+1}=v_{n} u_{i} v_{n}$. Hence there exists $v^{\prime}$ such that $v_{n} u_{i}=v^{\prime} a u$. Therefore $a=x_{s}$, where $s=\left|v^{\prime}\right|$. 
Since the tuple $\left(u_{0}, u_{1}, \ldots, u_{m-1}\right)$ generates the bi-ideal $x$ then $\exists k>n v_{k+1}=v_{k} u_{j} v_{k}$. Hence there exists $v^{\prime \prime}$ such that $v_{k} u_{j}=v^{\prime \prime} b u$. Therefore $b=x_{\sigma}$, where $\sigma=\left|v^{\prime \prime}\right|$. Since $k>n$ then $v_{n} \in \operatorname{Pref}\left(v_{k}\right)$. Thus $x_{s+t}=x_{\sigma+t}$ but $x_{s}=a \neq b=x_{\sigma}$. That means that $t$ is not a period of $x$.

(iii) Let us suppose that $T$ is a period of $x$. Then $\forall n \in \mathbb{Z}_{+} n T$ is a period too. This denies (i) and (ii) as well.

Corollary 7. Let $A$ be an alphabet and every letter $a \in A$ is chosen with one and the same probability $p(a)=\frac{1}{|A|}$. Let $p$ be a probability that a bi-ideal generated by $\left(u_{0}, u_{1}, \ldots, u_{m}\right)$ is ultimately periodic. If $\forall i\left|u_{i}\right| \geq n$ then $p \leq \frac{1}{A^{m n}}$.

Remarks. (i) Let $A=\{0,1\}$ and $m=n=10$ then probability $p \leq \frac{1}{2^{100}}$. This is practically negligible value.

(ii) Let a tuple $\left(u_{0}, u_{1}, \ldots, u_{m}\right)$ has been generated. Let $u$ be the longest word of this tuple. There is only one dubious situation by Theorem 6 if we like a bi-ideal that is not periodic. This happens if all words of the tuple $\left(u_{0}, u_{1}, \ldots, u_{m}\right)$ are prefixes and suffixes of $u$. This can be easy verified by deterministic algorithm. Thus we have indeed practical method how to generate a bi-ideal that is not periodic.

Corollary 8. If a bi-ideal generated by $\left(u_{0}, u_{1}, \ldots, u_{m-1}\right)$ is periodic then

$$
\forall i \forall j \quad\left(u_{i} \in \operatorname{Pref}\left(u_{j}\right) \cap \operatorname{Suff}\left(u_{j}\right) \vee u_{j} \in \operatorname{Pref}\left(u_{i}\right) \cap \operatorname{Suff}\left(u_{i}\right)\right) .
$$

Corollary 9. The class of periodic words $\mathfrak{P}$ is the proper subclass of the class of finitely generated bi-ideals $\mathfrak{B}_{\mathfrak{f}}$.

The following two lemmata are very easy, but those turn out to be extremely useful:

Lemma 10. If $x=w^{\omega}$ and $T$ is the minimal period of the word $x$, then $T \backslash|w|$, i.e. $T$ divides $|w|$.

Proof. Let $n=T|w|$, then both $T$ and $|w|$ are periods of the word $x[0, n)$. Hence [Theorem 1] $t=$ $\operatorname{gcd}(T,|w|)$ is a period of $x[0, n)$. Now we have

$$
\forall i x[0, n)=x[n i,(n+1) i)
$$

Therefore $t$ is a period of $x$. Since $T$ is the minimal period of the word $x$, then $t \geq T \geq \operatorname{gcd}(T,|w|)=t$. Hence $T=\operatorname{gcd}(T,|w|)$, thereby $T \backslash|w|$.

Lemma 11. If $x=w^{\omega}=u v y$ and $|w|=|v|$, then $v y=y=v^{\omega}$.

Proof. Let $|w|=t$ and $|u|=k+1$, then $v=x_{k+1} x_{k+2} \ldots x_{k+t}$, since $|v|=|w|$. We have $\forall i x_{i+t}=x_{i}$, therefore

$$
\forall j \in \overline{1, t} \forall s \quad x_{k+j}=x_{k+j+s t} .
$$

Example 12. The bi-ideal generated by $(0,010)$ is not periodic.

Proof. (i) Let $x$ be the bi-ideal generated by $(0,010)$, and

$$
\begin{aligned}
w_{0} & =0 \\
w_{1} & =00100 \\
w_{2} & =00100000100 \\
\cdot & \cdot \\
w_{2 n} & =w_{2 n-1} 0 w_{2 n-1} \\
w_{2 n+1} & =w_{2 n} 010 w_{2 n}
\end{aligned}
$$


in other words $x=\lim _{k \rightarrow \infty} w_{k}$.

Let $t$ be a period $\underset{k \rightarrow \infty}{\operatorname{kn}} x$. Then $t>3$, otherwise the period of $w_{2}$ must be less than or equal to 3 . Contradiction. So we have a word $w$ such that $|w|=t>3$ and $x=w^{\omega}$.

(ii) Now choose $n$ so large odd number that $t<\left|w_{n}\right|$. Then

$$
x=w_{n} 0 w_{n} \ldots
$$

and

$$
w_{n}=(u v)^{s} u,
$$

where $s \geq 1$, $u v=w$ and $u \neq \lambda$. (If $u=\lambda$ or $v=\lambda$, then $t$ divides $\left|w_{n}\right|$. We shall analyse this situation later.) From Lemma 11 we conclude that

$$
x=w_{n} 0 w^{\omega}=w_{n} 0(u v)^{\omega}=w_{n} 0 x .
$$

Thus

$$
(u v)^{s} \text { uvuvu } \ldots=(u v)^{s} u 0 u v u v \ldots
$$

Hence

$$
\text { vuvu... = 0uvuv } \ldots
$$

Since $u \neq \lambda$ and $u \in \operatorname{Pref}(x)$, then $u=0 u^{\prime}$. Hence

$$
v u \ldots=00 u^{\prime} v \ldots
$$

Thus, if $|v| \geq 2$, then $v=00 v^{\prime}$.

(iii) Note that

$$
w_{n} 0 x=x=w_{n} 0 w_{n} 010 w_{n} \ldots
$$

Therefore $x=w_{n} 010 w_{n} \ldots$ and

$$
(u v)^{\omega}=x=(u v)^{s} u 010 \ldots
$$

Hence $v=01 v^{\prime \prime}$ but $v=00 v^{\prime}$. Contradiction.

(iv) It remains to check that $|v| \leq 1$. Note

$$
u 010 \ldots=u v u \ldots
$$

Hence, if $|v|=1$, we can conclude that the first letter of $u$ is 1 . Contradiction! Otherwise $v=\lambda$, then $u=01 u^{\prime \prime}$. Again contradiction, since $w_{1}=00100$, therefore the first two letters of $u$ must be 00. Finally, if $u=\lambda$, then it remains to interchange $u$ with $v$ in the last two sentences of the proof.

Now turn our attention to Corollary 8 . We have proved that condition

$$
\forall i \forall j \quad\left(u_{i} \in \operatorname{Pref}\left(u_{j}\right) \cap \operatorname{Suff}\left(u_{j}\right) \vee u_{j} \in \operatorname{Pref}\left(u_{i}\right) \cap \operatorname{Suff}\left(u_{i}\right)\right)
$$

is necessary for periodicity of finitely generated bi-ideals. Nevertheless Example 12 demonstrates that this condition is not sufficient.

The following lemma is crucial:

Lemma 13. If a bi-ideal $x$ generated by $\left(u_{0}, u_{1}, \ldots, u_{m-1}\right)$ is periodic, then

$$
\forall i \forall j u_{i} x=u_{j} x .
$$


Proof. (i) Since $x$ is a bi-ideal generated by $\left(u_{0}, u_{1}, \ldots, u_{m-1}\right)$, then

$$
x=\lim _{k \rightarrow \infty} v_{k}
$$

$$
\begin{aligned}
& \text { where } \quad v_{0}=u_{0}, \quad v_{k+1}=v_{k} u_{k+1} v_{k} \text {, } \\
& \text { and } \quad u_{k+1}=u_{i} \text {, if } \quad k+1 \equiv i(\bmod m) \text {. }
\end{aligned}
$$

Let $t$ be a period of $x$ and choose $n$ so large that $t<\left|v_{n}\right|$. For every $i \in \overline{0, m-1}$ we can find $s_{i}>n$ such that

$$
v_{s_{i}+1}=v_{s_{i}} u_{i} v_{s_{i}} \text {. }
$$

Hence, by Corollary 2,

$$
\forall i \exists v_{i}^{\prime} v_{s_{i}}=v_{n} v_{i}^{\prime} v_{n}
$$

Therefore

$$
x=v_{s_{i}} u_{i} v_{s_{i}} \ldots=v_{n} v_{i}^{\prime} v_{n} u_{i} v_{n} \ldots
$$

(ii) We suppose that $x$ is periodic, thereby

$$
x=v^{\omega} \text {, where } v=x[0, t) .
$$

Note $v \in \operatorname{Pref}\left(v_{n}\right)$, therefore [Lemma 11]

$$
x=v_{n} v_{i}^{\prime} x=v_{n} v_{i}^{\prime} v_{n} u_{i} x .
$$

Hence, $\forall i x=v_{n} u_{i} x$, thereby $\forall i \forall j u_{i} x=u_{j} x$.

\section{Examples 14.}

(i) First, we reexamine Example 12 in light of the above lemma. Let us suppose that a bi-ideal $x$ generated by $(0,010)$ is periodic then $0 x=010 x$. This contradicts the fact that the first letter of $x$ is not 1 but 0 . The same arguments show that a bi-ideal generated by $(010,0)$ is not periodic too.

(ii) Both bi-ideals generated by $\left((01)^{n-1} 0,(01)^{n} 0\right)$ and $\left((01)^{n} 0,(01)^{n-1} 0\right)$ are not periodic. Indeed, if we suppose that a bi-ideal $x$ generated by $\left((01)^{n-1} 0,(01)^{n} 0\right)$ is periodic then by Lemma 13

$$
(01)^{n-1} 0 x=(01)^{n} 0 x=(01)^{n-1} 010 x .
$$

Hence $x=10 x$. This contradicts the fact that the first letter of $x$ is not 1 but 0 .

The same arguments show that a bi-ideal generated by $\left((01)^{n} 0,(01)^{n-1} 0\right)$ is not periodic too.

We now present some useful observations concerning the periodicity. We start with the following lemma.

Lemma 15. If $\exists u \in A^{+} u x=x \in A^{\omega}$, then a word $x$ is periodic with the minimal period $T \backslash|u|$.

Proof. Let $u=a_{1} a_{2} \ldots a_{t-1}$, where $\forall j a_{j} \in A$, and $y=u x$, then

$\forall i x_{i}=y_{i+t}$. Let

$$
y=u x=x .
$$

Hence

$$
\forall i y_{i}=x_{i}=y_{i+t} \text {. }
$$

This means that $y$ is periodic with a period $t$. Since $y=x$, then $x$ is periodic with a period $t$ too. Let $T$ is the minimal period of $x$, then by Lemma $10 T \backslash t$, i.e. $T \backslash|u|$.

Proposition 16. A word $x \in A^{\omega}$ is periodic if and only if

$$
\exists u \in A^{+} u x=x \in A^{\omega} .
$$


Proof. $\Rightarrow$ If $x$ is periodic then $\exists u \in A^{+} x=u^{\omega}$. Hence $x=u u^{\omega}=u x$. $\Leftarrow$ Lemma 15 .

Corollary 17. Let $u \in\left\{u_{0}, u_{1}, \ldots, u_{m-1}\right\}$ and $|u|=\max \left\{\left|u_{0}\right|,\left|u_{1}\right|, \ldots,\left|u_{m-1}\right|\right\}$. If $T$ is the minimal period of a periodic bi-ideal $x$ generated by $\left(u_{0}, u_{1}, \ldots, u_{m-1}\right)$ then $T<|u|$.

Proof. If only $u \neq \lambda$ then $u=u_{0}$ and $x=u^{\omega}$. Hence the minimal period $T<|u|$.

Otherwise, there exists $v \in\left\{u_{0}, u_{1}, \ldots, u_{m-1}\right\}$ such that $u \neq v \neq \lambda$. Now by Lemma $13 \quad u x=v x$. Hence

$$
\exists v^{\prime} \neq u \quad u=v v^{\prime} .
$$

Thus $v v^{\prime} x=v x$, therefore $v^{\prime} x=x$. Since $0<\left|v^{\prime}\right|$ then $T \backslash\left|v^{\prime}\right|$. This follows immediately from Lemma 15 . Thereby $T<\left|v^{\prime}\right|<|u|$.

Proposition 18. (see, e.g., [10]) Let $u, v \in A^{+}$be such that $u v=v u$. Then there exists $w \in A^{+}$such that $u, v \in w^{+}$.

Lemma 19. Let $u, v \in A^{+}$be such that $u^{k} v=v u^{k}$ for any positive integer $k$. Then there exists $w \in A^{+}$ such that $u, v \in w^{+}$.

Proof. If $k=1$ then it is Proposition 18. Now we assume that $k>1$; by Proposition 18

$$
\exists x \in A^{+}\left(u^{k}, v \in x^{+}\right) .
$$

(i) If $|x|>\left|u^{k-1}\right|$, then $x=u^{k}$, because $\left|x^{2}\right|>\left|u^{k}\right|$ and $u^{k} \in x^{+}$. Hence $x \in u^{+}$, therefore $v \in u^{+}$. Thus $\exists w \in A^{+}\left(u, v \in w^{+}\right)$; here $w=u$.

(ii) If $|x| \leq\left|u^{k-1}\right|$, then $l=\operatorname{gcd}(|x|,|u|)$ is period of $u^{k}$ by Theorem 1. Let

$$
w \in \operatorname{Pref}(u) \wedge|w|=l
$$

then $u \in w^{+}$. Since $u^{k} \in x^{+}$then $u^{k}=x^{m}$ for any $m$. Hence $x^{m} \in w^{+}$. Since $|w| \backslash|x|$ then $x \in w^{+}$. Therefore $v \in w^{+}$.

Theorem 20. If $x$ is a periodic bi-ideal generated by $\left(u_{0}, u_{1}\right)$ then

$$
\exists w u_{0}, u_{1} \in w^{*} .
$$

Proof. Obviously, if $u_{0}=u_{1}$ then a bi-ideal generated by $\left(u_{0}, u_{1}\right)$ is periodic. Now suppose that $u_{0} \neq u_{1}$. Then by Lemma $13 u_{0} x=u_{1} x$.

(i) Let $u_{0} \in \operatorname{Pref}\left(u_{1}\right)$, then $u_{1}=u_{0} u$, where $u \neq \lambda$, and $u_{0} x=u_{1} x=u_{0} u x$, therefore $x=u x$. Thus

$$
\begin{aligned}
x & =u_{0} u_{1} \ldots=u_{0} u_{0} u \ldots \\
x & =u x=u u_{0} u_{0} \ldots
\end{aligned}
$$

Hence $u_{0}^{2} u=u u_{0}^{2}$, and by Lemma $19 \exists w u_{0}, u_{1} \in w^{*}$.

(ii) Let $u_{1} \in \operatorname{Pref}\left(u_{0}\right)$, then $u_{0}=u_{1} u$, where $u \neq \lambda$, and $u_{0} x=u_{1} u x$. Since $u_{0} x=u_{1} x$ then $u_{1} u x=u_{1} x$, therefore $x=u x$. Thus

$$
\begin{aligned}
& x=u_{0} \ldots=u_{1} u \ldots \\
& x=u x=u u_{0} \ldots=u u_{1} u \ldots
\end{aligned}
$$

Hence $u_{1} u=u u_{1}$, and by Proposition $18 \exists w u, u_{1} \in w^{*}$. Since $u_{0}=u_{1} u$ then $u_{0} \in w^{*}$.

Theorem 21. A bi-ideal $x$ generated by $\left(u_{0}, u_{1}, \ldots, u_{m-1}\right)$ is periodic if and only if

$$
\exists w \forall i \in \overline{0, m-1} u_{i} \in w^{*} .
$$


Proof. Since $x$ is a bi-ideal generated by $\left(u_{0}, u_{1}, \ldots, u_{m-1}\right)$, then

$$
x=\lim _{k \rightarrow \infty} v_{k}
$$

$$
\begin{array}{rll}
\text { where } & v_{0}=u_{0}, & v_{k+1}=v_{k} u_{k+1} v_{k}, \\
\text { and } & u_{k+1}=u_{i}, & \text { if } \quad k+1 \equiv i(\bmod m) .
\end{array}
$$

$\Rightarrow$ We have $\quad u_{0} x=u_{1} x=\ldots=u_{m-1} x \quad$ by Lemma 13 .

(i) First, we shall prove that $\exists w u_{0}, u_{1} \in w^{*}$.

a) If $u_{1}=\lambda$ or $u_{1}=u_{0}$, then $w=u_{0}$.

Now we shall consider the situation $\lambda \neq u_{1} \neq u_{0}$.

b) Let $u_{0} \in \operatorname{Pref}\left(u_{1}\right)$ then

$$
\begin{aligned}
u_{1} & =u_{0} u, \quad \text { where } u \neq \lambda, \quad \text { and } \\
u_{0} x & =u_{1} x=u_{0} u x, \text { therefore } x=u x . \\
x & =u_{0} u_{1} \ldots=u_{0} u_{0} u \ldots \\
x & =u x=u u_{0} u_{0} \ldots
\end{aligned}
$$

Hence $u_{0}^{2} u=u u_{0}^{2}$, and by Lemma $19 \exists w u_{0}, u \in w^{*}$. Since $u_{1}=u_{0} u$ then $u_{1} \in w^{*}$.

c) Let $u_{1} \in \operatorname{Pref}\left(u_{0}\right)$ then

$$
\begin{aligned}
u_{0} & =u_{1} u, \quad \text { where } u \neq \lambda, \quad \text { and } \\
u_{1} x & =u_{0} x=u_{1} u x, \text { therefore } x=u x . \\
x & =u_{0} \ldots=u_{1} u \ldots \\
x & =u x=u u_{0} \ldots=u u_{1} \ldots
\end{aligned}
$$

Hence $u_{1} u=u u_{1}$, thereby [Proposition 18] $\exists w u, u_{1} \in w^{*}$. Since $u_{0}=u_{1} u$, then $u_{0} \in w^{*}$.

(ii) Further, we shall prove the theorem by induction on $n$, i.e., suppose that $\exists v \forall i \in \overline{0, n} \quad u_{i} \in v^{*}$. Let $u_{n} \neq u_{n+1} \neq \lambda$, otherwise $u_{n+1} \in v^{*}$.

a) Let $u_{n} \in \operatorname{Pref}\left(u_{n+1}\right)$ then

$$
\begin{aligned}
u_{n+1} & =u_{n} u, \quad \text { where } \quad u \neq \lambda, \quad \text { and } \\
u_{n} x & =u_{n+1} x=u_{n} u x, \text { therefore } x=u x . \\
x & =v_{n} u_{n+1} \ldots=v_{n} u_{n} u \ldots \\
x & =u x=u v_{n} u_{n} \ldots
\end{aligned}
$$

Hence $v_{n} u_{n} u=u v_{n} u_{n}$. We have by induction $\exists k v^{k}=v_{n} u_{n}$ and $k \geq 1$, since $u_{0} \neq \lambda$. Thus $v^{k} u=u v^{k}$, and by Lemma $19 \exists w v, u \in w^{*}$.

Thereby $v \in w^{*}$, and by induction $\forall i \in \overline{0, n} \quad u_{i} \in v^{*}$. Hence $\forall i \in \overline{0, n} u_{i} \in w^{*}$. Since $u_{n+1}=u_{n} u$ and $u_{n}, u \in w^{*}$, then $u_{n+1} \in w^{*}$.

b) Let $u_{n+1} \in \operatorname{Pref}\left(u_{n}\right)$ then

$$
\begin{aligned}
u_{n} & =u_{n+1} u, \quad \text { where } u \neq \lambda, \quad \text { and } \\
u_{n+1} x & =u_{n} x=u_{n+1} u x, \text { therefore } x=u x . \\
x & =v_{n-1} u_{n} \ldots=v_{n-1} u_{n+1} u \ldots \\
x & =u x=u v_{n-1} u_{n+1} \ldots
\end{aligned}
$$


Hence $v_{n-1} u_{n+1} u=u v_{n-1} u_{n+1}$, therefore by Proposition $18 \exists w_{0} v_{n-1} u_{n+1}, u \in w_{0}^{*}$. We have by induction $\exists k v^{k}=v_{n-1}, k \geq 1$, since $u_{0} \neq \lambda$. Thus

$$
\left|v_{n-1} u_{n}\right|=\left|v_{n-1} u_{n+1} u\right|>\left|v_{n-1}\right|+|u| \geq|v|+\left|w_{0}\right|
$$

and $v_{n-1} u_{n} \in v^{*}, v_{n-1} u_{n}=v_{n-1} u_{n+1} u \in w_{0}^{*}$. This means that both $|v|$ and $\left|w_{0}\right|$ are periods of $v_{n-1} u_{n}$. Now by Theorem $1 l=\operatorname{gcd}\left(|v|,\left|w_{0}\right|\right)$ is the period of $v_{n-1} u_{n}$. Let

$$
w \in \operatorname{Pref}\left(v_{n-1}\right) \wedge|w|=l
$$

then $v_{n-1} u_{n} \in w^{+}$because $l \backslash\left|v_{n-1} u_{n}\right|$.

The word $v_{n-1} u_{n}=u_{i_{1}} u_{i_{2}} \ldots u_{i_{\varkappa}}$, where all $u_{i_{s}} \in\left\{u_{0}, u_{1}, \ldots, u_{n}\right\}$, besides,

$$
\forall j \in \overline{0, n} \exists \nu \in \overline{1, \varkappa} \quad u_{j}=u_{i_{\nu}} .
$$

Since $\forall i \in \overline{0, n} u_{i} \in v^{*}$ then $\forall i \in \overline{0, n} l \backslash\left|u_{i}\right|$. Thus $\forall i \in \overline{0, n} u_{i} \in w^{*}$.

Finally, $u_{n}=u_{n+1} u$ and $u_{n} \in w^{*}$, therefore $l$ is the period of $u_{n+1}$. Since $u \in w_{0}^{*}$ then $l \backslash|u|$. Hence $l \backslash\left|u_{n+1}\right|$. Thus $u_{n+1} \in w^{*}$.

This completes the induction.

$\Rightarrow$ Since $\forall n v_{n} \in w^{*}$ then $x=w^{\omega}$.

\section{The Class of Bounded Bi-ideals}

Definition 22. Let $\left(u_{i}\right)$ generates a bi-ideal $x$. The bi-ideal $x$ is called bounded if $\exists l \forall i\left|u_{i}\right| \leq l$.

Proposition 23. The class of finitely generated bi-ideals $\mathfrak{B}_{\mathfrak{f}}$ is the proper subclass of the class of bounded bi-ideals $\mathfrak{B}_{\mathfrak{b}}$.

Proof. Note $\operatorname{card}\left\{\left(u_{i}\right) \mid \forall i u_{i} \in\{0,1\}\right\}=\mathfrak{c}$ - the cardinality of the set of real numbers. Let $\left(u_{i}\right)$, $\left(v_{i}\right)$ be two different sequences of letters in the alphabet $\{0,1\}$ that generate bi-ideals $\left(x_{i}\right),\left(y_{i}\right)$ respectively. Since $\left(x_{i}\right) \neq\left(y_{i}\right)$ then card $\left\{\left(x_{i}\right) \mid\right.$ there is a sequence $\left(u_{i}\right)$ of letters in the alphabet $\{0,1\}$ that generate a bi-ideal $\left.\left(x_{i}\right)\right\}=\operatorname{card}\left\{\left(u_{i}\right) \mid \forall i u_{i} \in\{0,1\}\right\}=\mathfrak{c}$.

Let $\mathfrak{U}_{m}=\left\{\left(u_{0}, u_{1}, \ldots, u_{m-1}\right) \mid \forall i u_{i} \in\{0,1\}^{*}\right\}$ then card $\bigcup_{m=1}^{\infty} \mathfrak{U}_{m}=\aleph_{0}$, where $\aleph_{0}$ is the first infinite cardinality. Therefore the cardinality of the set of all finitely generated bi-ideals in the alphabet $\{0,1\}$ is equal to $\aleph_{0}$. Since $\aleph_{0}<\mathfrak{c}$ then $\mathfrak{B}_{\mathfrak{f}} \subset \mathfrak{B}_{\mathfrak{b}}$.

Let $w=u_{1} w_{1} v_{1}=u_{2} w_{2} v_{2}$. We define $a$ meet $w_{1} \cap w_{2}$ as follows. If there exists an occurrence $\left(u_{3}, w_{3}, v_{3}\right)$ of $w_{3}$ in word $w$ such that $w=u_{3} w_{3} v_{3}$, where $\left|u_{3}\right|=\max \left(\left|u_{1}\right|,\left|u_{2}\right|\right),\left|v_{3}\right|=\max \left(\left|v_{1}\right|,\left|v_{2}\right|\right)$, then $w_{1} \cap w_{2}=w_{3}$. Otherwise, $w_{1} \cap w_{2}=\lambda$.

Lemma 24. Let $\left(u_{i}\right)$ generates a bi-ideal $x, v_{0}=u_{0}, \forall i\left(v_{i+1}=v_{i} u_{i+1} v_{i}\right)$ and $\forall i\left|u_{i}\right| \leq l$. If $v \in \mathrm{F}(x)$ and $|v|=2\left|v_{m}\right|+l$ for some $m$, then $v_{m} \in \mathrm{F}(v)$.

Proof. Since $v \in \mathrm{F}(x)$ then $v \in \mathrm{F}\left(v_{n}\right)$ but $v \notin \mathrm{F}\left(v_{n-1}\right)$ for some $n$. Moreover, $v_{n-1} u_{n} v_{n-1}=v_{n}=v^{\prime} v v^{\prime \prime}$ for some $v^{\prime}, v^{\prime \prime}$.

Since $v \notin \mathrm{F}\left(v_{n-1}\right)$ then $u_{n} \cap v \neq \lambda$. Hence, $\left|v_{n-1} \cap v\right| \geq\left|v_{m}\right|$ because of $\left|u_{n}\right| \leq l$. Therefore $v_{m} \in \mathrm{F}(v)$ by Corollary 2 .

Definition 25. It is said a factor $u$ of an infinite word $x$ occurs syndetically in $x$ if there exists an integer $k$ such that in any factor of $x$ of length $k$ there is at least one occurrence of $u$. A word $x$ is called uniformly recurrent, or with bounded gaps, when all its factors occur syndetically in $x$.

Proposition 26. Bounded bi-ideals are uniformly recurrent. 
Proof. Let $x$ be a bounded bi-ideal generated by $\left(u_{i}\right)$ then there exists $l$ such that $\forall i\left|u_{i}\right| \leq l$. Let $u \in \mathrm{F}(x), v_{0}=u_{0}$ and $\forall i\left(v_{i+1}=v_{i} u_{i+1} v_{i}\right)$ then there exists $m$ such that $u \in \mathrm{F}\left(v_{m}\right)$.

Let $v \in \mathrm{F}(x)$ and $|v|=2\left|v_{m}\right|+l$ then $v_{m} \in \mathrm{F}(v)$ by Lemma 24. Therefore $u \in \mathrm{F}(v)$. So the factor $u$ of $x$ occurs syndetically in $x$.

Let $\phi: A^{*} \rightarrow A^{*}$ be a nonerasing morphism (namely, $\phi\left(A^{+}\right) \subseteq A^{+}$) such that there exists a letter $a \in A$ such that

For all $n \geq 0$ one has

$$
\phi(a)=a u, \quad \text { with } \quad u \in A^{+}
$$

$$
\phi^{n+1}(a)=\phi^{n}(a u)=\phi^{n}(a) \phi^{n}(u)
$$

so that $\phi^{n}(a)$ is a proper prefix of $\phi^{n+1}(a)$. Thus the sequence $\left(\phi^{n}(a)\right)$ converges to a limit denoted by $\phi^{\omega}(a)$, that is,

$$
\phi^{\omega}(a)=\lim _{n \rightarrow \infty} \phi^{n}(a) .
$$

One says that $x=\phi^{\omega}(a)$ is the infinite word obtained by iterating the morphism $\phi$ on the letter a. Moreover, one has $x=\phi(x)$, that is, $x$ is a fixed point for $\phi$.

Very famous infinite word is Thue-Morse word $t$ on two letters

$$
t=0110100110010110 \ldots
$$

t can be introduced by iterating, on the letter 0 , the morphism

$$
\tau:\{0,1\}^{*} \rightarrow\{0,1\}^{*}, \quad \text { defined as } \tau(0)=01, \tau(1)=10 .
$$

The word $t$ was introduced by Thue in two papers [15, 16] of 1906 and 1912 and, subsequently, rediscovered by Morse [11] and several other authors. Thue-Morse word is uniformly recurrent (see, e.g., [10]).

Definition 27. A factor $u$ of a word $x \in A^{\infty}$ is called an overlapping factor of $x$ if $u=$ avava, with $a \in A$ and $v \in A^{*}$. We say that $x$ is overlap-free, if $x$ does not contain overlapping factors.

Corollary 28. Let $y \in A^{\infty}$. If $x \backslash y$ and $x=$ uvuvu, where $u \neq \lambda$, then both $x$ and $y$ contain an overlapping factor.

Proof. Since $u \neq \lambda$, then exist a letter $a \in A$ and a word $w \in A^{*}$ such that $u=a w$. Hence $x=$ $(a w) v(a w) v(a w)=a(w v) a(w v) a w$. Thus $x$ contains the overlapping factor $a(w v) a(w v) a$.

Proposition 29. (see, e.g., [10]) The Thue - Morse word $t$ is overlap-free.

Lemma 30. If $\stackrel{\infty}{\forall i} u_{i}=\lambda$ then a bi-ideal $x$ generated by $\left(u_{i}\right)$ is periodic.

Proof. Let $v_{0}=u_{0}, \quad v_{i+1}=v_{i} u_{i+1} v_{i}$. Since $\stackrel{\infty}{\forall} i u_{i}=\lambda$ then $\exists n \forall i>n u_{i}=\lambda$. Hence $v_{n+1}=v_{n} u_{n+1} v_{n}=v_{n}^{2}$.

Further, we shall prove the lemma by induction on $j$, i.e., suppose that $v_{n+j}=v_{n}^{k}$, where $k=2^{j}$, then $v_{n+j+1}=v_{n+j} u_{n+j+1} v_{n+j}=v_{n+j}^{2}=v_{n}^{2 k}$, where $2 k=2 \cdot 2^{j}=2^{j+1}$.

Thus $x=\lim _{i \rightarrow \infty} v_{i}=\lim _{k \rightarrow \infty} v_{n}^{k}=v_{n}^{\omega}$.

Lemma 31. If $A$ is a finite alphabet then every bounded bi-ideal $x \in A^{\omega}$ contains an overlapping factor.

Proof. Let $x \in A^{\omega}$ be a bi-ideal generated by the sequence $u_{0}, u_{1}, \ldots, u_{n}, \ldots$

(i) If $\stackrel{\infty}{\forall} \quad i \quad u_{i}=\lambda$ then $x$ is periodic [Lemma 30]. Therefore [Corollary 28] $x$ contains an overlapping factor.

(ii) If $\stackrel{\infty}{\exists} i u_{i} \neq \lambda$, then $\exists i \exists j\left(i<j \wedge u_{i}=u_{j}\right)$, because $A$ is finite and $\exists l \forall i\left|u_{i}\right| \leq l$. Since $x$ is the bi-ideal generated by the sequence $u_{0}, u_{1}, \ldots, u_{n}, \ldots$ then $x=\lim _{n \rightarrow \infty} v_{n}$, where $v_{0}=u_{0}$ and $v_{n+1}=v_{n} u_{n+1} v_{n}$. Hence by Corollary $2 v_{j}=v_{j-1} u_{j} v_{j-1}=v^{\prime} u_{i} v_{i-1} u_{j} v_{i-1} u_{i} v^{\prime \prime}$. Thus $u_{i} v_{i-1} u_{j} v_{i-1} u_{i} \backslash x$, therefore [Corollary 28] $x$ contains an overlapping factor. 
Proposition 32. The Thue-Morse word $t$ is not a bounded bi-ideal.

Proof. Let us suppose that $t$ is a bounded bi-ideal generated by $\left(u_{i}\right)$ then by Lemma $31 t$ contains an overlapping factor. This is contradiction [Proposition 29].

Theorem 33. The class of bounded bi-ideals $\mathfrak{B}_{\mathfrak{b}}$ is the proper subclass of the class of uniformly recurrent words $\mathfrak{R}_{\mathfrak{u}}$, that is, $\mathfrak{B}_{\mathfrak{b}} \subseteq \mathfrak{R}_{\mathfrak{u}}$ and $\mathfrak{B}_{\mathfrak{b}} \neq \mathfrak{R}_{\mathfrak{u}}$.

Proof. The class of bounded bi-ideals $\mathfrak{B}_{\mathfrak{b}}$ is the subclass of the class of uniformly recurrent words $\mathfrak{R}_{\mathfrak{u}}$ by Proposition 26. The Thue-Morse word is uniformly recurrent as mentioned above. Therefore $\mathfrak{B}_{\mathfrak{b}} \neq \mathfrak{R}_{\mathfrak{u}}$ by Proposition 32 .

Example 34. Let $x$ be the bi-ideal generated by $\left(u_{i}\right)$, where

$$
\begin{aligned}
u_{0} & =0 \\
u_{1} & =1 \\
\forall i>1 \quad u_{i} & =00100 .
\end{aligned}
$$

Then

$$
\begin{aligned}
& v_{0}=0 \\
& v_{1}=010 \\
& v_{2}=01000100010 \\
& v_{3}=010001000100010001000100010
\end{aligned}
$$

and $x=\lim _{i \rightarrow \infty} v_{i}$. Thus $x$ is the bounded bi-ideal, besides $x=(0100)^{\omega}$. This demonstrates that straightforward generalization of Theorem 21 for bounded bi-ideals is not valid.

Convention Let $x$ be a bi-ideal generated by $\left(u_{i}\right)$, then $x=\lim _{i \rightarrow \infty} v_{i}$, where $v_{0}=u_{0}$ and $v_{i+1}=v_{i} u_{i+1} v_{i}$. We adopt this notational convention henceforth.

Lemma 35. If $v_{n} u \in v^{*}$ and $\forall i \in \mathbb{Z}_{+} u_{n+i} \in u v^{*}$, then

$$
\forall i \in \mathbb{N} v_{n+i} \in v^{*} v_{n} .
$$

Proof. If $i=0$ then $v_{n+i}=v_{n}=\lambda v_{n} \in v^{*} v_{n}$.

Further, we shall prove the lemma by induction on $i$, i.e., suppose that $v_{n+i} \in v^{*} v_{n}$, namely,

$$
\exists k \in \mathbb{N} v_{n+i}=v^{k} v_{n} .
$$

By assumption, $v_{n} u \in v^{*}$ and $u_{n+i+1} \in u v^{*}$, i.e.

$$
\exists l \in \mathbb{N} v_{n} u=v^{l} \wedge \exists m \in \mathbb{N} u_{n+i+1}=u v^{m} .
$$

Hence

$$
\begin{aligned}
v_{n+i+1} & =v_{n+i} u_{n+i+1} v_{n+i}=\left(v^{k} v_{n}\right)\left(u v^{m}\right)\left(v^{k} v_{n}\right) \\
& =v^{k}\left(v_{n} u\right) v^{m+k} v_{n}=v^{k} v^{l} v^{m+k} v_{n} \in v^{*} v_{n}
\end{aligned}
$$

This completes the induction. 
Lemma 36. If $t$ is the period of the bi-ideal $x$ and $\left|v_{n}\right| \geq t$, then

$$
\forall i \in \mathbb{Z}_{+} u_{n+1} x=u_{n+i} x .
$$

Proof. We have $v_{n+i}=v_{n+i-1} u_{n+i} v_{n+i-1}$. Hence, if $i \in \mathbb{Z}_{+}$then [Corollary 2]

$$
\forall i \in \mathbb{Z}_{+} \exists v_{i}^{\prime} v_{n+i}=v_{n} v_{i}^{\prime} v_{n} .
$$

Now, by definition of $x$

$$
\begin{aligned}
x & =v_{n} u_{n+1} v_{n} \ldots \\
x & =v_{n+i} u_{n+i+1} v_{n+i} \ldots=v_{n} v_{i}^{\prime} v_{n} u_{n+i+1} v_{n} \ldots
\end{aligned}
$$

By assumption, $x$ is periodic, therefore

$$
x=v^{\omega}, \quad \text { where } \quad|v|=t .
$$

Since $v \in \operatorname{Pref}\left(v_{n}\right)$ then by Lemma 11

$$
\begin{aligned}
& x=v_{n} u_{n+1} x, \\
& x=v_{n} u_{n+i+1} x .
\end{aligned}
$$

Hence $\forall i \in \mathbb{Z}_{+} x=v_{n} u_{n+i} x$. Thus $\forall i \in \mathbb{Z}_{+} u_{n+1} x=u_{n+i} x$.

Theorem 37. A bi-ideal $x$ is periodic if and only if

$$
\exists n \in \mathbb{N} \exists u \exists v\left(v_{n} u \in v^{*} \wedge \forall i \in \mathbb{Z}_{+} u_{n+i} \in u v^{*}\right) .
$$

$\Rightarrow$ Let $T$ be the minimal period of the word $x$, then $\exists n \in \mathbb{N}\left|v_{n}\right| \geq T$. Thus by Lemma 36

$$
\forall i \in \mathbb{Z}_{+} \quad u_{n+1} x=u_{n+i} x .
$$

Let $u$ be the longest word of the set $\bigcap_{i=1}^{\infty} \operatorname{Pref}\left(u_{n+i}\right)$ then

$$
\forall i \in \mathbb{Z}_{+} \exists u_{i}^{\prime}\left(u_{n+i}=u u_{i}^{\prime}\right) .
$$

Particularly, $\exists k u_{n+k}=u$. This means that

$$
\forall i \in \mathbb{Z}_{+} \quad u u_{i}^{\prime} x=u_{n+i} x=u_{n+k} x=u x .
$$

Thus

Hence by Lemma 15

$$
\forall i \in \mathbb{Z}_{+} \quad u_{i}^{\prime} x=x
$$

$$
\forall i \in \mathbb{Z}_{+} \quad T \backslash\left|u_{i}^{\prime}\right|
$$

Thereby

where $v=x[0, T)$. Thus

$$
\forall i \in \mathbb{Z}_{+} \quad u_{i}^{\prime} \in v^{*}
$$$$
\forall i \in \mathbb{Z}_{+} \quad u_{n+i}=u u_{i}^{\prime} \in u v^{*}
$$

Note

$$
x=v_{n} u_{n+1} v_{n} \ldots=v_{n} u u_{1}^{\prime} v_{n} \ldots
$$

Since $u_{1}^{\prime} \in v^{*}$ and $v \in \operatorname{Pref}\left(v_{n}\right)$, then [Lemma 11] $x=v_{n} u x$. Hence [Lemmma 15] $v_{n} u \in v^{*}$. $\Leftarrow$ By Lemma 35

$$
\forall i \in \mathbb{N} \exists k_{i} \in \mathbb{N} v_{n+i}=v^{k_{i}} v_{n} .
$$

Since $\lim _{k \rightarrow \infty}\left|v_{k}\right|=\infty$ then $\lim _{i \rightarrow \infty} k_{i}=\infty$. Thus

$$
x=\lim _{k \rightarrow \infty} v_{k}=\lim _{i \rightarrow \infty} v_{n+i}=\lim _{i \rightarrow \infty} v^{k_{i}} v_{n}=v^{\omega} .
$$




\section{References}

[1] D. B. Bean, A. E. Ehrenfeucht and G. McNulty. (1979) Avoidable Patterns in Strings of Symbols. Pacific J. Math. 85, 261-294.

[2] J. Berstel, J. Karhumäki. (2003) Combinatorics on Words - A Tutorial. TUCS Technical Report (No 530, June).

[3] M. Coudrain and M. P. Schützenberger. (1966) Une condition de finitude des monö̈des finiment engendrés. C. R. Acad. Sc. Paris, Sér. A, 262, 1149-1151.

[4] M. Crochemore and W. Rytter. (1995) Squares, cubes, and time-space efficient string searchinng. Algorithmica 13, 405-425.

[5] N.J. Fine, H.S. Wilf. (1965) Uniqueness Theorem for Periodic Functions. Proc. Amer. Math. Soc. 16, 109114.

[6] D. Gusfield. (1997) Algorithms on Strings, Trees, and Sequences. Cambridge University Press.

[7] N. Jacobson. (1964) Structure of Rings. American Mathematical Society, Providence, RI.

[8] M. Lothaire. (1983) Combinatorics on Words. Encyclopedia of Mathematics and its Applications, Vol. 17, Addison-Wesley, Reading, Massachusetts.

[9] M. Lothaire. (2002) Algebraic Combinatorics on Words. Encyclopedia of Mathematics and its Applications, Vol 90, Cambridge University Press, Cambridge.

[10] Aldo de Luca, Stefano Varricchio. (1999) Finiteness and Regularity in Semigroups and Formal Languages. Springer-Verlag, Berlin, Heidelberg.

[11] M. Morse. (1921) Recurrent geodesics on a surface of negative curvature. Trans. Amer. Math. Soc. 22, 84-110.

[12] R. A. Rueppel. (1986) Analysis and Design of Stream Ciphers. Springer-Verlag, Berlin.

[13] I. Simon. (1988) Infinite Words and a Theorem of Hindman. Rev. Mat. Apl. 9, 97-104.

[14] J. A. Storer. (1988) Data compression: methods and theory. Computer Science Press, Rockville, MD.

[15] A. Thue (1906) Über unendliche Zeichenreihen. Norske Vidensk. Selsk. Skrifter. I. Mat.-Nat. K1., Christiania Nr 7, 1-22.

[16] A. Thue (1912) Über die gegenseitige Lage gleicher Teile gewisser Zeichenreihen. Norske Vidensk. Selsk. Skrifter. I. Mat.-Nat. K1., Christiania Nr 10, 1-67.

[17] А. И. Зимин. (1982) Блокирующие множества термов. [Blocking Sets of Terms.] Матем. сб., т.119, № 3 , с. $363-375$. (Russian) 
